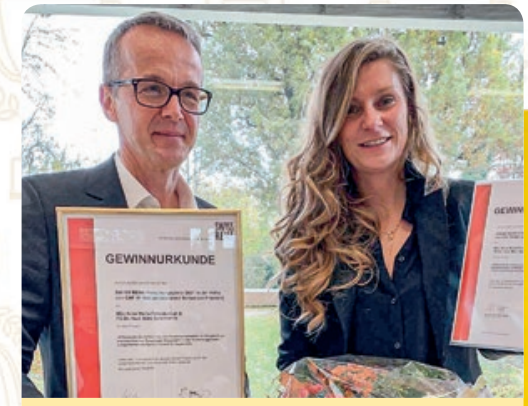

PD Dr. med. Marc Spielmanns, Chefarzt Pulmonale Rehabilitation und Ärztlicher Direktor der Zürcher RehaZentren und MSc Anna Maria Pekacka-Egli, Leitung Logopädie der Zürcher RehaZentren, Klinik Wald (@ Swiss REHA).

\section{SW!SS REHA} Forschungspreis

Der Verband der führenden Schweizer Rehabilitationskliniken SW!SS REHA verleiht erstmals in seiner Geschichte den Forschungspreis der Schweizer Rehabilitation. Der Preis geht an ein Forschungsteam der Klinik Wald der Zürcher RehaZentren unter Leitung von MSc Anna Maria Pekacka-Egli und PD Dr. med. Marc Spielmanns für ein Forschungsprojekt zur Vorhersage von Lungenentzündungen nach einem Schlaganfall. Der Preis in der Höhe von CHF 10000 soll künftig alle zwei Jahre vergeben werden, um ein wissenschaftliches Projekt auf dem Gebiet der Rehabilitationsforschung in der Schweiz auszuzeichnen.

\section{Prix Label 2021 de la Fondation Dalle Molle}

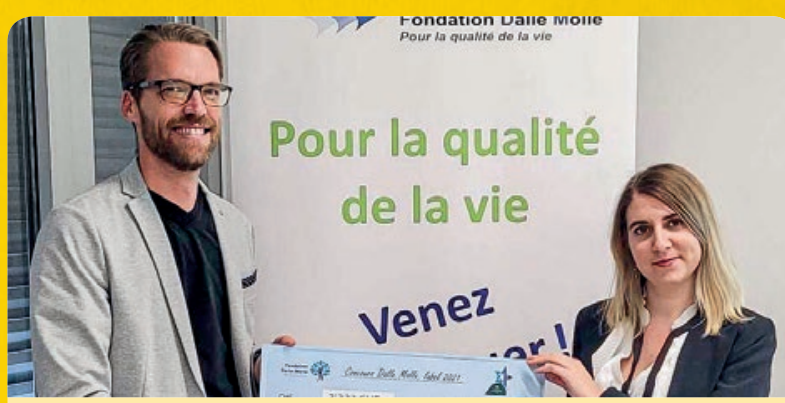

Frédéric Ehrler et Laetitia Gosetto (๔ Frédéric Erhler / HUG).

Le Prix Label 2021 pour la qualité de la vie a été remis au projet PELOSHA (Personalizable Services For Supporting Healthy Aging). Il est décerné par la Fondation Dalle Molle à but non lucratif, basée à Lugano.

Mené par Laetitia Gosetto du Service des sciences de l'information médicale et Frédéric Ehrler de la Direction des systèmes d'information des Hôpitaux universitaires de Genève, le projet PELOSHA vise à favoriser le maintien des personnes âgées à domicile par le biais de deux applications. L'une d'elles permet aux personnes âgées d'activer des modules selon leurs besoins (suivi de santé, du sommeil, de l'activité physique). Les informations de ces modules sont envoyées au personnel soignant via smartphone. La seconde application consiste en un tableau de bord destiné au personnel soignant qui joue le rôle de centre de contrôle et d'évaluation de la qualité de vie et de la santé des personnes âgées.

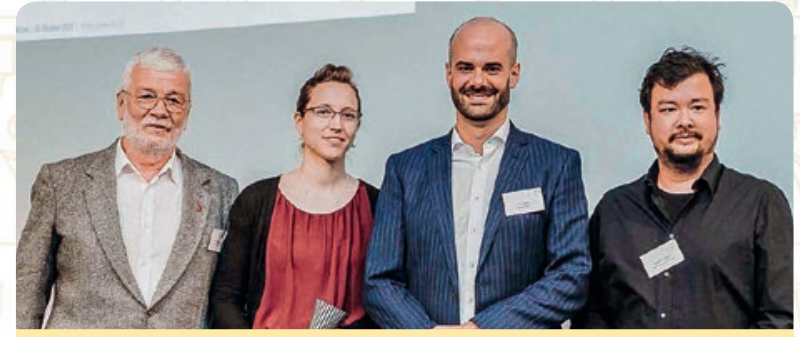

(v.l.n.r.) Dr. med. Emil Schalch, Verwaltungsratspräsident, Gesundheitspunkt Oberägeri; Andrea Barmet, Leitende Medizinische Praxiskoordinatorin und Mitglied der Geschäftsleitung, Gesundheitspunkt Oberägeri; Urs Vögeli, santeneXt; Fabian Schalch, Sozialarbeiter, Gesundheitspunkt Oberägeri (๔ santeneXt).

\section{Prix d'excellence santeneXt}

Der Gesundheitspunkt Oberägeri wird mit dem Prix d'excellence santeneXt für sein Versorgungsmodell ausgezeichnet. Die enge Zusammenarbeit mit den Patientinnen und Patienten sowie eine lückenlose elektronische Dokumentation zeichnen die Versorgungsqualität des Zuger Gesundheitspunkts Oberägeri aus. «Unsere Patientinnen und Patienten sind Leader ihres Teams und lassen sich auf ihrem Weg beraten», sagt Dr. med Emil Schalch, Initiator und Leiter des Projektes. Mit dem Prix d'excellence zeichnet der von Galenica, Interpharma und Swica getragene Do Tank santeneXt jährlich ein innovatives Kollaborationsprojekt mit Modellcharakter für das Schweizer Gesundheitswesen aus.

\section{Deux Prix en ophtalmologie}

Le SWISSophthAWARD et le Swiss VitreoRetinal Group (SVRG) ont récompensé la Dre Benedetta Franceschiello et le Dr Lorenzo Di Sopra pour leurs travaux sur l'imagerie par résonance magnétique sur l'œil en mouvement. Lors des examens de l'œil, il est souvent compliqué d'obtenir une image qui puisse servir de diagnostic, car de nombreuses personnes ont de la peine à garder leurs yeux immobiles suffisamment longtemps.

Leurs recherches leur ont valu le prix de la «Plus grande pertinence clinique» du SWISSophthAWARD. Décerné par Bayer Schweiz AG, ce prix récompense des scientifiques travaillant dans le domaine du système visuel. Le SRVG les a distingués de son Prix Retina, sponsorisé par Novartis Pharma Suisse AG et attribué à de jeunes chercheuses et chercheurs pour des travaux dans le domaine de la rétine médicale majoritairement effectués en Suisse.
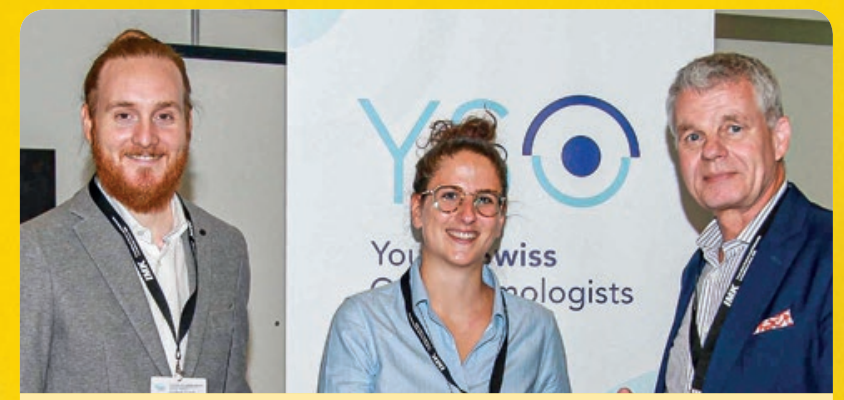

De g. à dr. Dr Lorenzo Di Sopra, Benedetta Franceschiello et Prof. Dr Christian Pruente (๔ Société suisse d'ophtalmologie). 